\title{
Perforating Folliculitis in Pregnancy: A Case Report
}

\author{
Pandey $\mathrm{D}^{1}$ and Garg $\mathrm{D}^{2 *}$ \\ ${ }^{1}$ Department of Obstetrics and Gynaecology, Vardhman Mahavir Medical College and \\ Safdarjung hospital, India \\ 2Department of Obstetrics and Gynaecology, NDMC Medical College and Hindu Rao \\ Hospital, India
}

Short Communication

Volume 2 Issue 1

Received Date: March 22, 2018

Published Date: April 02, 2018

DOI: $10.23880 /$ whsj-16000109

*Corresponding author: Deepali Garg, Department of Obstetrics And Gynaecology, NDMC Medical College and Hindu Rao Hospital, India, Tel: 9818706660; Email: drdeepaligarg@gmail.com

\section{Abstract}

Perforating Folliculitis (PF) classified amongst Acquired Perforating Dermatoses can lead to preterm delivery especially in presence of secondary infection. This entity should be kept in mind as a potential cause of preterm labor in females with itchy skin lesion with papulo-vesiculo-pustular lesions.

Keywords: Acquired Perforating Dermatoses; APLA syndrome; Preterm labour

Abbreviations: PF: Perforating Folliculitis; RPL: Recurrent Pregnancy Loss; APLA: Anti phospholipid antibody; KP: Keratosis Pilari; EPS: Elastosis Perforans Serpigina

\section{Introduction}

Perforating disorders consist of heterogenous group defined by trans-epidermal extrusion of dermal substances. Perforating Folliculitis (PF) is characterized by peri-follicular granulomatous process along with destruction of pilary complex and occasional perforation of hair through the infundibulum $[1,2]$.

It is characterized by asymptomatic skin coloured or erythematous aggregated or scattered follicular papules with a central keratotic plug mainly affecting the limbs but may also present on the trunks, buttocks and even the scalp and face [3].

Diseases associated with PF have included psoriasis, Juvenile Acanthosis Nigricans, hypertension and atherosclerotic cardiovascular disorders, Diabetes Mellitus, Chronic Renal Failure and HIV infected human [4-9].
We hereby present a case of perforating folliculitis during pregnancy where the patient had a preterm delivery. Perforating folliculitis has not been reported so far in pregnancy.

\section{Case History}

A 25 years old P0L0A3 (all first trimester losses) presented to gynecology Outpatient department for work up of recurrent pregnancy loss (RPL). She had APLA syndrome (Anti phospholipid antibody) [diagnosed during RPL work up at our centre] and thus was put on low molecular weight heparin and low dose aspirin since the start of her pregnancy. She took regular antenatal visits and underwent all routine pregnancy investigations which were normal. Her last visit was 3 weeks back. Her pregnancy progressed well till 32 weeks when she presented to our casualty with threatened preterm labour. On examination patient had multiple follicular papules over the extensor surface with insidious onset, associated with itching and increasing gradually over to involve the lower extremities and buttocks. Occasional pustules were also seen Figure 1. Per abdomen examination showed single live intra-uterine fetus of 32 weeks with cephalic 


\section{Women's Health Science Journal}

presentation with mild contractions. Patient received steroid cover for fetal lung maturity. Patient's high vaginal swab and urine culture and sensitivity showed no growth, liver function tests were normal. Dermatological consultation was sought and she was given anti-histaminics. Local steroid were not given due to presence of occasional pustules. The lesion as well as the associated itching did not respond. So after 1 week of treatment, she underwent biopsy from skin lesion. She was given antibiotic ampicillin in view of her pustules (after collecting culture report of pus which showed growth of staphylococcus sensitive to ampicillin). But the patient went in spontaneous preterm labour and delivered $1.8 \mathrm{~kg}$ male baby at $33+2$ weeks. The histopathology report was collected postpartum, showed Perforating Folliculitis. The lesions and pruritis gradually resolved in post-delivery period. The patient was discharged along with the baby after 2 weeks.

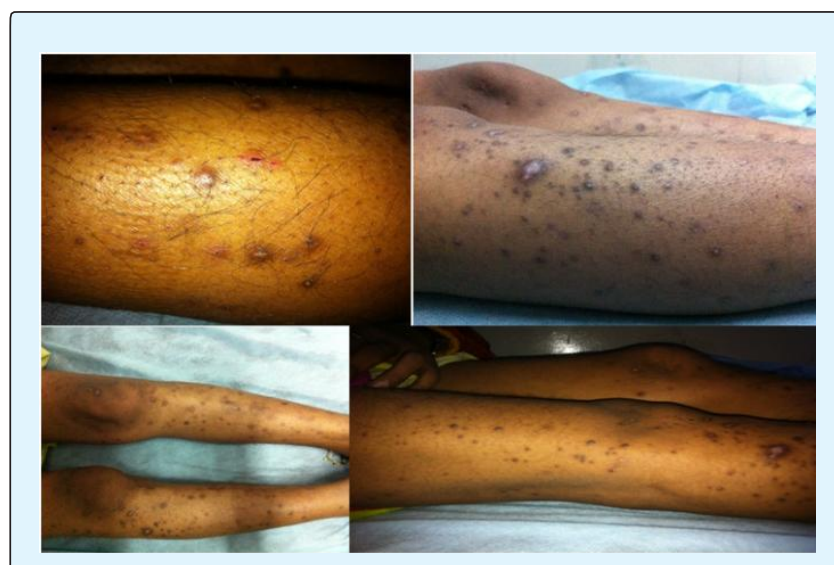

Figure 1: Photograph of skin lesions (papules and pustules) present on extensor surface of lower limbs of a pregnant female whose biopsy proved to be Perforating Folliculitis.

\section{Discussion}

The entity of perforating folliculitis is classified under Acquired perforating dermatoses. On thorough literature search using the MeSH terms "perforating folliculitis" and "pregnancy" on pubmed from 1900 to 2018, we could not retrieve any case. Only one report, which was though not of perforating folliculitis but about the entity of Acquired Perforating dermatoses (which refers to condition with altered collagen and elastin fibres that undergo trans-epidermal elimination), has been reported in a pregnant female [4]. But in that case no underlying etiological factor was found. Healy et al suggested pruritis as the main contributing cause [4].

Most patients belong to $2^{\text {nd }}$ to $4^{\text {th }}$ decade with equal sex involvement. This entity has unknown aetiology
$[5,6]$. However, mechanical disruption of follicular epithelium by hair is said to play role. Sometimes these hair are not always found even after careful sectioning [7].

The location of PF is on extensor surfaces of the extremities highlights the role of low grade chronic friction which incites abnormal keratinization within the follicular infundibulum ultimately causing the perforation of epithelium and exposure of dermis to follicular center. The trapped hair usually hastens the process. In our patient also, the location of the lesions was on extensor surface of lower extremity.

The inflammatory and necrotic substance is finally eliminated through the perforating canal by means of trans-epidermal elimination. There may be a yet undetected connective tissue change as an inciting event [5]. In Diabetes, microangiopathy can be the main inciting event. Clinically these lesions resemble other folliculitis like bacterial and fungal folliculitis and acne vulgaris. Culture studies and distribution pattern can help to distinguish. We started ampicillin in our patient considering it bacterial folliculitis and due to presence of pustules. But our patient did not respond.

A close entity is Keratosis Pilari (KP) which has same clinical resemblance to $\mathrm{PF}$ in both lesion type and location. If multiple biopsies are taken for KP, occasional perforating follicle can be demonstrated, implying PF to be a phenomenon rather than a specific disease. However in PF, perforating process dominates the clinical scenario [6].

This entity has been reported in psoriasis, Juvenile Acanthosis Nigricans, hypertension and atherosclerotic cardiovascular disorders, Diabetes Mellitus, Chronic Renal Failure and HIV infected human [5-9]. Histopathologically, it needs to be distinguished from entities like Elastosis Perforans Serpigina (EPS), Reactive perforating collagenosis and Keratosis Pilaris.

The unusual part in our case is the pregnancy which precipitated the condition probably due to immunocompromised state partly due to pregnancy itself and partly secondary to auto-immune disease. The super added infection probably provided a septic focus and led to preterm delivery in the patient. We should have started antibiotic empirically for pustules without waiting for culture reports.

\section{Conclusion}

Dermatoses like perforating folliculitis can lead to preterm birth especially in event of secondary infection. This entity should be kept in mind as a potential cause of preterm labor in females with itchy skin lesion. 


\section{References}

1. Bardach H (1979) Folliculitis granulamtousperfor an sprofunda: an independent perforating dermatosis? Hautarzt 30: 489-493.

2. Patterson JW (1984) The perforating disorders. J Am Acad Dermatol 10(4): 561-581.

3. Saray Y, Seckin D, Bilezikci B (2006) Acquired perforating dermatosis: Clinicopathological features in twenty two cases. J Eur. Acad Dermatol Venereol 20(6): 679-688.

4. Healy R, Cerio R, Hollingsworth A, Bewley A (2010) Acquired perforating dermatosis associated with pregnancy. Clin Exp Dermatol 35(6): 621-623.

5. Mehregan AH, Coskey RJ (1968) Perforating folliculitis. Arch Dermatol 97(4): 394-399.
6. Mohd El Sayed, Adel AL, Abdul Razzaq, Salim Alotaibi (2003) Perforating Dermatoses-Review Article. The Gulf Journal of Dermatology 10(2): 1422.

7. Patterson JW, CraffJ W, Eubanks SW (1982) Perforating folliculitis and Psoriasis. J Am Acad Dermatol 7(3): 369-376.

8. Hurwitz RM (1985) The evolution of perforating folliculitis in patients with chronic renal failure. Am J Dermatopathol 7(3): 231-239.

9. Rubio FA, Herranz P, Robayna G, Pena JM, Contreras F, et al. (1999) Perfotating Folliculitis, report of a case in an HIV infected man. J Am Dermatol 40(2): 300-302.

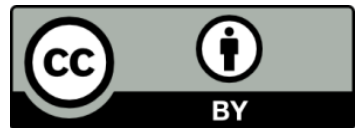

